\title{
The Key Role of Staging Definitions for Assessment of Downstaging for Hepatocellular Carcinoma
}

\author{
Francis Y. Yao, MD ${ }^{1}$ Nicholas Fidelman, MD ${ }^{2} \quad$ Neil Mehta, MD ${ }^{1}$ \\ ${ }^{1}$ Division of Gastroenterology, Department of Medicine, University of \\ California, San Francisco \\ 2 Division of Interventional Radiology, Department of Radiology, \\ University of California, San Francisco \\ Address for correspondence Francis Y. Yao, MD, Division of \\ Gastroenterology, Department of Medicine, University of California, \\ San Francisco, 513 Parnassus Avenue, Room S-357, San Francisco, CA \\ 94143-0538 (e-mail: francis.yao@ucsf.edu).
}

Semin Liver Dis 2021;41:117-127.

\begin{abstract}
Keywords

- hepatocellular carcinoma

- downstaging

- liver transplant

- local regional therapy

- $\alpha$-fetoprotein

The success of liver transplant (LT) for hepatocellular carcinoma (HCC) is dependent on accurate tumor staging using validated imaging criteria, and adherence to acceptable criteria based on tumor size and number. Other factors including $\alpha$-fetoprotein (AFP) and response to local regional therapy (LRT) have now played a larger role in candidate selection. Tumor downstaging is defined as reduction in the size of viable tumors using LRT to meet acceptable criteria for LT, and serves as a selection tool for a subgroup of HCC with more favorable biology. The application of tumor downstaging requires a structured approach involving three key components in tumor staging-initial tumor stage and eligibility criteria, tumor viability assessment following LRT, and target tumor stage prior to LT-and incorporation of AFP into staging and treatment response assessments. In this review, we provide in-depth discussions of the key role of these staging definitions in ensuring successful outcome.
\end{abstract}

More than two decades ago, the Milan criteria (1 nodule $\leq 5 \mathrm{~cm}$ or $2-3$ nodules $\leq 3 \mathrm{~cm}$ ) defined the benchmark for achieving survival after liver transplant (LT) in hepatocellular carcinoma (HCC) similar to that of nonmalignant indications. ${ }^{1}$ As a result, LT has been widely accepted as an effective treatment for early-stage HCC with an acceptable risk for post-LT HCC recurrence using these restrictive criteria. ${ }^{2-5}$ Over time, however, we have witnessed a gradual shift in the approach to patient selection. Rather than relying solely on the morphologic criteria based on tumor size and number, we have incorporated surrogates of tumor "biology" metrics, most notably $\alpha$-fetoprotein (AFP) and tumor response to local regional therapy (LRT), in the selection scheme. ${ }^{3-7}$ Downstaging of HCC represents a structured approach that aims at merging expansion of tumor size limits beyond conventional criteria with objective and sustained response to LRT as an additional risk stratification tool. ${ }^{8}$ It is believed that response to LRT with successful downstaging of HCC is a marker of a more favorable tumor biology in a subset of patients with initial tumor burden beyond conventional LT criteria who would likely also do well after LT. $^{8}$ As data on downstaging continue to emerge, ${ }^{8,9}$ this strategy has gained broader acceptance in clinical practice. ${ }^{4-6}$ While many other criteria have been proposed for LT beyond the Milan criteria, with most combining an assessment of tumor burden with AFP, ${ }^{10-15}$ downstaging has recently been incorporated into the organ allocation policy for HCC in the United States. Defining the initial tumor stage prior to downstaging, treatment end point with respect to tumor stage prior to LT, and how to assess tumor stage following LRT are key components of the downstaging process. In this review, we emphasize the key roles of these staging definitions in helping to ensure successful outcome of LT after tumor downstaging.

\section{Structured Approach to Tumor Downstaging}

A highly structured and multidisciplinary approach is essential in the successful application of downstaging treatments and in the assessments of treatment response over time. - Fig. 1 summarizes the essential steps in the execution of tumor downstaging, which includes three key components related to tumor staging-initial tumor stage and eligibility criteria, published online

January 14, 2021 (c) 2021. Thieme. All rights reserved. Thieme Medical Publishers, Inc., 333 Seventh Avenue, 18th Floor, New York, NY 10001, USA
DOI https://doi.org/ 10.1055/s-0040-1716565. ISSN 0272-8087. 


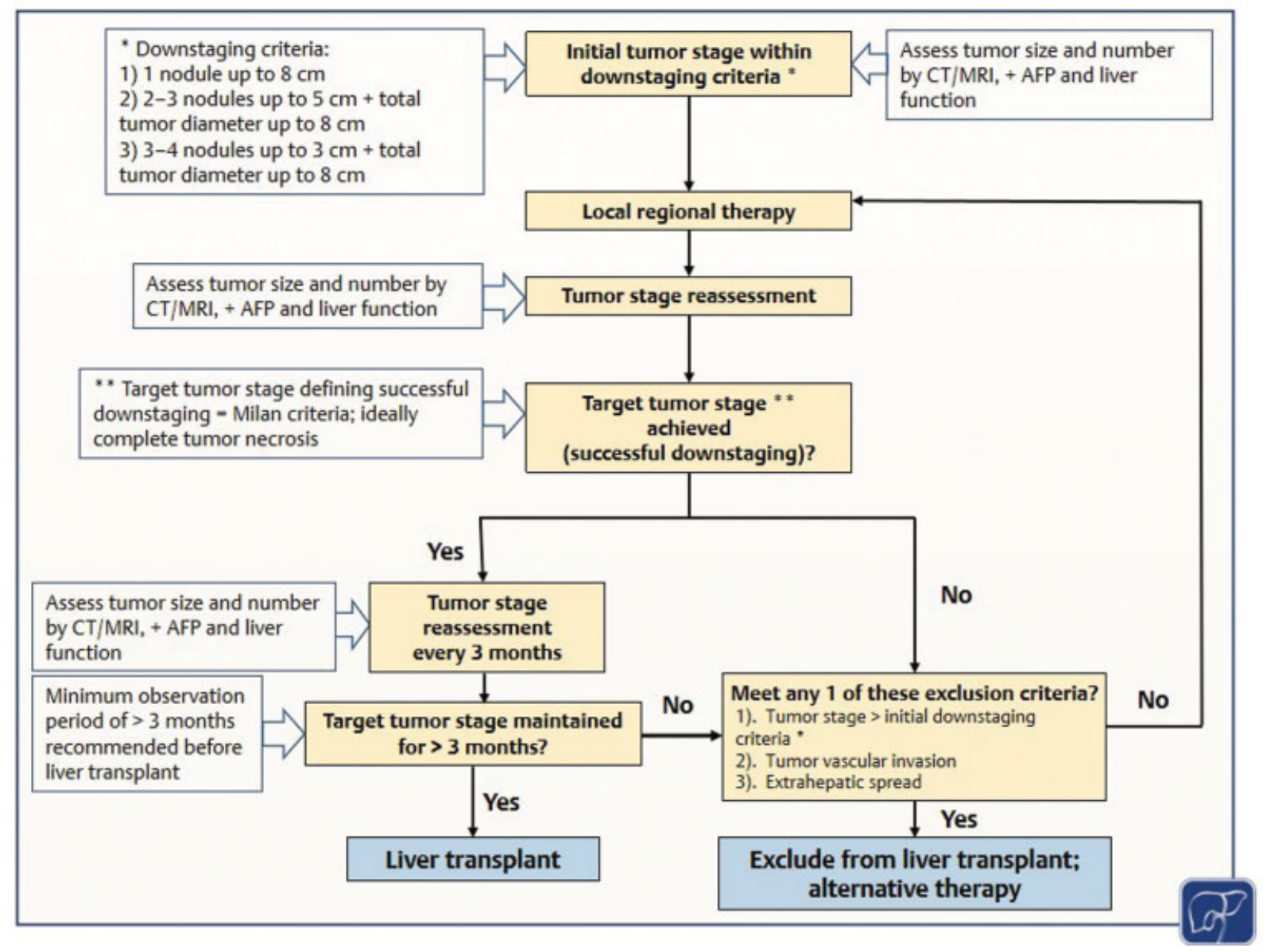

Fig. 1 Schematic diagram of downstaging protocol.

tumor viability assessment following LRT, and target tumor stage (treatment end point) prior to LT. AFP is an important prognostic marker and should be incorporated into staging and treatment response assessments. A minimal observation period of 3 months from successful tumor downstaging to LT without additional LRT treatment is recommended to ensure disease stability prior to $\mathrm{LT} .^{8,16}$ We define treatment failure and exclusion from LT as tumor progression to beyond entry criteria for downstaging, tumor vascular invasion, or extrahepatic metastases. It is important to emphasize that careful radiologic assessments are needed not only for tumor burden in the liver, but also to exclude extrahepatic disease or tumor vascular invasion at baseline and at every time point in the staging and re-evaluation process. The proposed staging definitions are summarized in -Table $\mathbf{1}$. All the important components related to tumor staging will be discussed in detail in the subsequent sections in this review.

\section{Radiologic Assessment of HCC Diagnosis and Stage}

The diagnosis of HCC is established radiographically if typical imaging features are present on a multiphase dynamic contrast-enhanced computed tomography (CT) or magnetic resonance imaging (MRI) in patients with cirrhosis or other known risk factors for HCC. The presence of arterial hyperenhance- ment followed by washout on delayed phases of imaging is highly specific for HCC $\geq 10 \mathrm{~mm}$ in diameter, and these diagnostic parameters have been adopted by the American Association for the Study of Liver Disease (AASLD) since 2005 and the European Association for the Study of Liver disease (EASL) practice guidelines. ${ }^{2,3}$

The Liver Imaging Reporting and Data System (LI-RADS) was launched in March 2011 to improve standardization and consensus regarding performance, interpretation, and reporting CT and MRI of the liver in patients with cirrhosis or other risk factors, ${ }^{17}$ and was also incorporated into the United Network for Organ Sharing (UNOS)/Organ Procurement and Transplantation Network (OPTN) guidelines. ${ }^{18}$ One of the goals of LI-RADS is to render false-positive imaging diagnoses of HCC as exceedingly rare. LI-RADS category assignment depends on four imaging criteria: arterial phase hyperenhancement, washout, capsule, and threshold growth (defined as > 50\% diameter increase in $<6$ months). Two of these criteria, namely capsule and threshold growth, have not been validated. A major concern using threshold growth in combination with arterial phase enhancement in radiologic diagnosis is the inability to differentiate between HCC and intrahepatic cholangiocarcinoma, which is increasing in prevalence among patients with cirrhosis and considered a contraindication to LT. ${ }^{19,20}$ As a result, radiologic diagnosis of HCC should be based on the AASLD 2005 or EASL criteria of arterial hyperenhancement 
Table 1 Standardized downstaging protocol

Eligibility criteria and initial tumor stage $^{\text {a }}$
1. UCSF downstaging criteria (UNOS/OPTN criteria)
(a) Single nodule $\leq 8 \mathrm{~cm}$
(b) 2 or 3 nodules each $\leq 5 \mathrm{~cm}$ with the sum of the maximal diameters of all nodules $\leq 8 \mathrm{~cm}$
(c) 3 or 4 nodules each $\leq 5 \mathrm{~cm}$ with the sum of the maximal diameters of all nodules $\leq 8 \mathrm{~cm}$

2. Absence of extrahepatic disease and vascular invasion on cross-sectional imaging of the chest and abdomen

3. Child's $A$ and $B$ cirrhosis with total bilirubin $\leq 3 \mathrm{mg} / \mathrm{dL}^{\mathrm{b}}$

Tumor staging after local regional therapy ${ }^{a}$

1. Only viable tumor(s) are considered; tumor-diameter measurements should not include the area of necrosis from local regional therapy

2. If there are more than one area of residual tumor enhancement, then the diameter of the entire lesion should be counted toward the overall tumor burden

3. When enhancement at or near the treatment site is considered equivocal for HCC recurrence, close follow-up with repeat imaging in 2-3 months is needed to ensure stability and absence of radiologic features to suggest HCC recurrence

4. If tumor progression beyond LT criteria occurs but the active tumor burden remains within downstaging entry criteria, LT should be put on hold, and repeat LRT should be undertaken until the end point of downstaging is achieved for the patients to be eligible again for LT

Target tumor stage prior to liver transplant (criteria for successful downstaging) ${ }^{a}$

1. Minimal criteria should be residual viable tumor size and diameter meeting Milan criteria

2. Complete tumor necrosis recommended prior to LT

Criteria for downstaging failure and exclusion from liver transplant ${ }^{a}$

1. Progression of tumor(s) to beyond inclusion/eligibility criteria for downstaging (as defined above)

2. Tumor invasion of a major hepatic vessel based on cross-sectional imaging of the abdomen

3. Lymph node involvement by tumor or extrahepatic spread of tumor based on cross-sectional imaging of the chest and abdomen (and additional imaging as clinically indicated)

4. AFP $\geq 1,000 \mathrm{ng} / \mathrm{mL}$; unless the AFP level decreases to $<100 \mathrm{ng} / \mathrm{mL}$ after local regional therapy

Additional guidelines

1. A minimal observation period of 3 months between successful tumor downstaging and liver transplant is required. This should be based on two consecutive cross-sectional imaging studies at least 3 months apart showing disease stability with successful downstaging being maintained according to imaging criteria without local regional therapy

2. A patient with acute hepatic decompensation after downstaging treatment is not eligible for liver transplant unless criteria for successful downstaging and minimal observation period are met

3. A patient with Child's B or C cirrhosis and AFP $\geq 1,000 \mathrm{ng} / \mathrm{mL}$ at baseline should not undergo downstaging ${ }^{c}$

Abbreviations: AFP, $\alpha$-fetoprotein; HCC, hepatocellular carcinoma; LT, liver transplant; OPTN, Organ Procurement and Transplantation Network; UCSF, University of California San Francisco; UNOS, United Network for Organ Sharing.

${ }^{a}$ Criteria based on cross-sectional imaging with multiphase CT or MRI of the abdomen showing arterial phase hyperenhancement and washout in the delayed phase for diagnosis of HCC.

${ }^{\mathrm{b}}$ Recommendations based on guidelines for transarterial chemoembolization procedure.

${ }^{\mathrm{C}}$ Based on $100 \%$ downstaging failure rate in these patients in one study. ${ }^{18}$

followed by washout on delayed phases, ${ }^{2,3}$ and biopsy of the lesion is indicated to provide histologic confirmation if the nodule does not fulfill the typical radiologic features of HCC.

Following LRT, radiographic assessments of tumor size should be based on measurements of the maximum diameter of only viable tumors by multiphase CT or MRI, and should not include the area of necrosis resulting from LRT. This follows the principles of the modified Response Evaluation Criteria in Solid Tumors (mRECIST) ${ }^{21}$ and EASL ${ }^{3}$ guidelines. More recently, LI-RADS provided further classifications based on tumor viability following LRT (see under tumor response following LRT).

While imaging criteria for the diagnosis of the main tumor nodule are based on validated criteria, controversies exist for the registration of additional/satellite nodules as HCC in the staging process. ${ }^{22}$ We apply the same criteria of arterial enhancement and washout in the classification of these additional nodules as HCC, and yet other additional lesions may be misclassified and contribute to tumor understaging. Consequently, further investigations based on clinicopathologic correlations are needed.

\section{Defining the Initial Tumor Stage to Qualify for Downstaging}

Restricting the upper limits in initial tumor burden (size and number) for downstaging serves two purposes. First, this approach avoids futile attempts, taking into consideration the low probability of achieving successful tumor downstaging in patients with very large tumors or a high number of tumor nodules, and the potential risks of LRT including hepatic decompensation. Second, it is likely that by relaxing the eligibility criteria, post-LT survival would be compromised even if downstaging is achieved prior to LT.

Only a few studies on tumor downstaging for LT have welldefined upper limits in tumor size and number at entry. ${ }^{8,9}$ In 


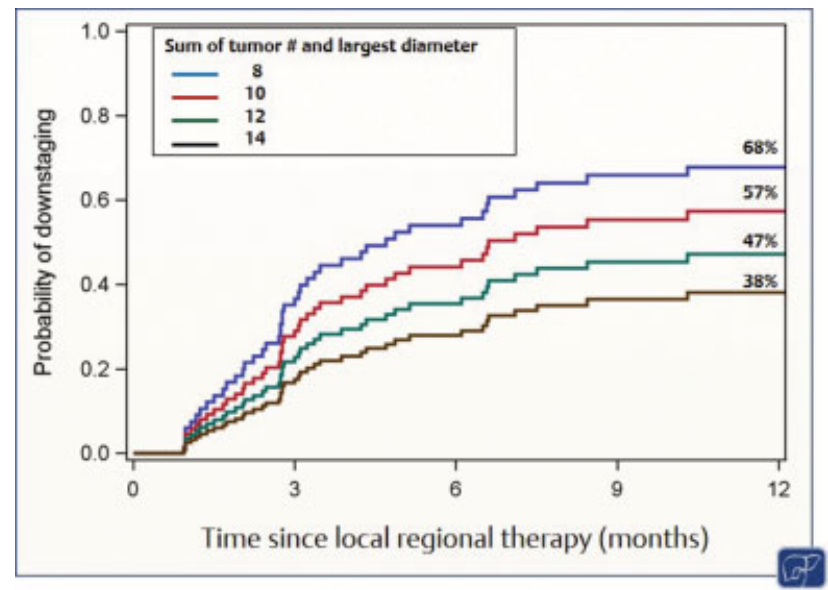

Fig. 2 The association between the probability of downstaging to Milan criteria and the initial tumor burden based on the sum of the largest tumor diameter and the number of tumor nodules. (Modified with permission from Reig et al. ${ }^{22}$ )

the University of California San Francisco (UCSF) downstaging protocol, ${ }^{23}$ the eligibility criteria included 1 nodule $\leq 8 \mathrm{~cm}, 2$ to 3 nodules each $\leq 5 \mathrm{~cm}$ and the sum of the maximal diameters $\leq 8 \mathrm{~cm}$, and 4 to 5 nodules each $\leq 3 \mathrm{~cm}$ and the sum of the maximal diameters $\leq 8 \mathrm{~cm}$. These tumor burden thresholds were modified from the proposed UCSF criteria, ${ }^{10}$ which was in part based on the observation that the total tumor diameter $>8 \mathrm{~cm}$ predicted worse survival after LT. In a study by Ravaioli et al from Bologna, Italy, ${ }^{24}$ the upper limits included 1 nodule $\leq 6 \mathrm{~cm}, 2$ nodules each $\leq 5 \mathrm{~cm}$, and 3 to 5 nodules each $\leq 4 \mathrm{~cm}$ with the sum of maximal diameters $\leq 12 \mathrm{~cm}$. Using Milan criteria as the end point of downstaging, $65 \%$ of patients in the UCSF study achieved successful downstaging and 54\% had received LT. In a subsequent multicenter study by Mehta et al applying the UCSF downstaging protocol, the probability of successful downstaging was $83 \%$ and $58 \%$ had received LT. $^{25}$ In the Bologna study, ${ }^{24} 67 \%$ of their patients were able to be downstaged to Milan criteria and underwent LT. Murali et $\mathrm{al}^{26}$ reported a significantly lower chance for successful downstaging in patients with initial tumor size and number exceeding UCSF criteria ${ }^{10}$ or the up-to- 7 criteria ${ }^{11}$ when compared with those with tumors within these criteria.

In other studies without clearly defined upper limits in the tumor burden, the proportion of successful downstaging was only $44 \%$, with a range of 11 to $77 \%{ }^{9}$ Sinha et al recently compared the outcomes of downstaging between the group meeting the UCSF downstaging criteria at entry versus those exceeding these criteria without upper limits in tumor size or number ("all-comers"). ${ }^{27}$ Successful downstaging to Milan was observed in $65 \%$ of the "all-comers" group versus $84 \%$ of those in the UCSF downstaging group. The "all-comers" group also had a significantly lower LT rate (14 vs. 59\%). This study also observed a good correlation between the sum of tumor number and largest tumor diameter and the likelihood of successful downstaging (-Fig. 2). The cumulative probability of successful downstaging at 1 year from time of first LRT decreased incrementally with a greater sum of the tumor number and largest tumor diameter. It went from $68 \%$ for those with a sum of 8 , to $57 \%$ in patients with this sum equal to $10,47 \%$ in patients with the sum of 12 , and $38 \%$ in those with a sum of 14 (- Fig. 3 ). These observations support the notion that an upper limit in tumor burden exists beyond which successful LT after tumor downstaging becomes an unrealistic goal. ${ }^{27}$

Post-LT survival following successful tumor downstaging to Milan criteria has been shown to be similar to that in patients meeting Milan criteria at the outset. The vast majority of these studies were based on initial tumor burden

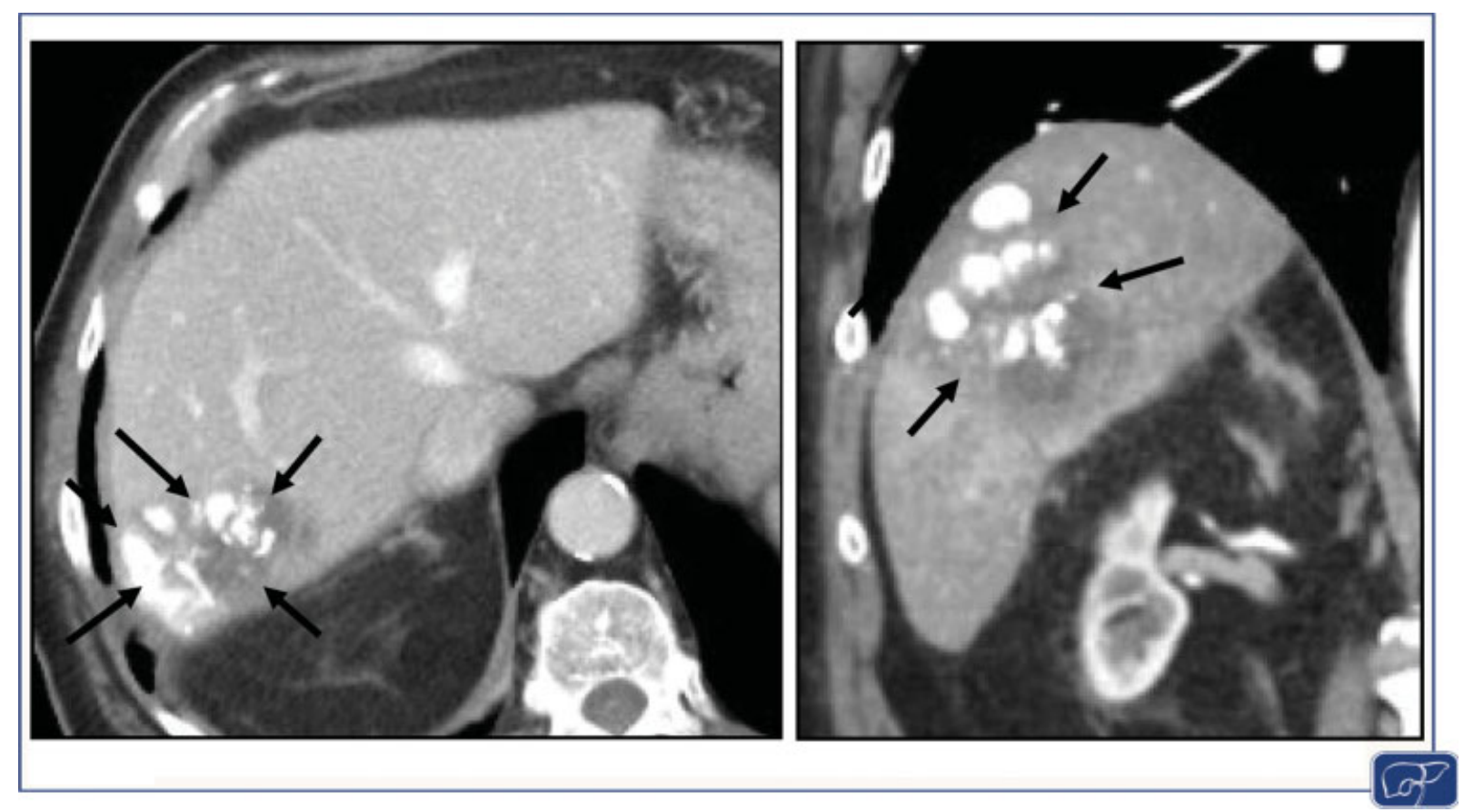

Fig. 3 Multiphase CT following conventional transarterial chemoembolization showing deposition of ethiodized oil (arrows) within the treated lesion. Subtle residual enhancement may be obscured by the chemoembolization material. CT, computed tomography. 
meeting the UCSF downstaging criteria, ${ }^{23,25,28,29}$ with 5-year post-LT survival up to $80 \%$ in the downstaged group. ${ }^{23,25}$ In the Bologna study, ${ }^{24}$ the 3 -year disease-free survival was lower at $71 \%$ but comparable to the group meeting Milan criteria at the outset. In a preliminary report by Tabrizian et al of the largest U.S. multicenter study to date, ${ }^{29}$ the 10 -year post-LT survival was $52 \%$ for the 330 patients with initial tumor stage meeting the UCSF downstaging criteria who achieved successful downstaging to Milan criteria, versus $61 \%$ among the 2,086 patients with tumors always within Milan. In contrast, the 10-year post-LT survival of the 110 patients with tumors beyond Milan criteria at LT was significantly lower at $39 \% .{ }^{29}$ To improve on the quality of this and other multicenter cohort studies on downstaging, radiologic staging criteria should be vigorously defined, prospectively implemented, and ideally based on an independent central review process to ensure accuracy. The post-LT outcomes from these studies using specific criteria for initial tumor burden are summarized in -Table 2 .

Whether the initial tumor stage prior to downstaging predicts post-LT outcome is still a matter of debate. A recent study by Mehta et $\mathrm{al}^{28}$ on the national experience of tumor downstaging using the UNOS database observed similar 3-year postLT survival among HCC patients always within Milan criteria (83\%) compared with those initially meeting UCSF downstaging criteria (79\%) who were successfully downstaged into Milan criteria prior to LT. However, the 3-year post-LT survival in those with initial tumor stage beyond these criteria despite successful downstaging was significantly lower at $71 \%{ }^{28} \mathrm{~A}$ prospective multicenter study is ongoing to address this issue.

As many LT centers began to employ tumor downstaging strategies for LT, staging definitions and end points varied widely across regions. ${ }^{30}$ In an effort to standardize criteria for downstaging, a new liver allocation policy has been implemented in the United States that would allow priority listing for LT for those with initial tumor burden meeting the UCSF downstaging criteria who have achieved successful downstaging to within Milan criteria. ${ }^{30}$ Applying these upper limits of initial tumor burden represents a starting point in the standardized application of tumor downstaging on a broader scale, and provides the opportunity for future validation and further refinements.

Table 2 Summary of posttransplant outcome following downstaging based on specific initial tumor staging criteria

\begin{tabular}{|c|c|c|c|c|}
\hline $\begin{array}{l}\text { Author, year, } \\
\text { institution } \\
\text { (reference) }\end{array}$ & $\begin{array}{l}\text { Initial tumor } \\
\text { staging } \\
\text { criteria }\end{array}$ & $\begin{array}{l}\text { Study } \\
\text { design }\end{array}$ & Post-transplant survival & p-Value \\
\hline $\begin{array}{l}\text { Yao 2015; } \\
\text { San Francisco, CA }{ }^{16}\end{array}$ & $\begin{array}{l}\text { UCSF } \\
\text { downstaging } \\
\text { criteria }\end{array}$ & $\begin{array}{l}\text { Prospective } \\
\text { single-center } \\
\text { study }\end{array}$ & $\begin{array}{l}\text { Downstaged ( } n=64 \text { ): } 5 \text {-year sur- } \\
\text { vival } 78 \% \text {; } 5 \text {-year recurrence-free } \\
\text { probability } 91 \% \\
\quad \text { Milan criteria (no downstaging) } \\
\text { ( } n=332 \text { ): } 5 \text {-year survival } 81 \%, 5 \text {-year } \\
\text { recurrence-free probability } 88 \%\end{array}$ & NS \\
\hline $\begin{array}{l}\text { Mehta 2018; } \\
\text { Multicenter } \\
\text { Region } 5 \text { ( } 3 \text { centers) } \\
18\end{array}$ & $\begin{array}{l}\text { UCSF } \\
\text { downstaging } \\
\text { criteria }\end{array}$ & $\begin{array}{l}\text { Retrospective } \\
\text { multicenter } \\
\text { study }\end{array}$ & $\begin{array}{l}\text { Downstaged }(n=109) \text { : } 5 \text {-year sur- } \\
\text { vival } 80 \% \text {; 5-year recurrence-free } \\
\text { probability } 87 \%\end{array}$ & \\
\hline $\begin{array}{l}\text { Mehta } 2020 ; \\
\text { UNOS database } 23\end{array}$ & $\begin{array}{l}\text { UCSF } \\
\text { downstaging } \\
\text { criteria }\end{array}$ & $\begin{array}{l}\text { Retrospective } \\
\text { database } \\
\text { analysis }\end{array}$ & $\begin{array}{l}\text { Within UCSF downstaging criteria } \\
\text { and downstaged to Milan }(n=422) \text { : } \\
\text { 3-year survival 79\% } \\
\text { Milan criteria (no downstaging) } \\
\text { ( } n=3,276) \text { : 3-year survival } 83 \% \\
\text { Beyond UCSF downstaging criteria, } \\
\text { and downstaged to Milan criteria } \\
(n=121): 3 \text {-year survival } 71 \%\end{array}$ & $\begin{array}{l}p=0.04 \text { for beyond UCSF } \\
\text { downstaging criteria vs. } \\
\text { Milan } \\
p=\text { NS for meeting UCSF } \\
\text { downstaging criteria vs. } \\
\text { Milan }\end{array}$ \\
\hline $\begin{array}{l}\text { Tabrizian } 2019 ; \\
\text { US multicenter } \\
\text { study ( } 5 \text { centers) }\end{array}$ & $\begin{array}{l}\text { UCSF } \\
\text { downstaging } \\
\text { criteria }\end{array}$ & $\begin{array}{l}\text { Retrospective } \\
\text { multicenter } \\
\text { study }\end{array}$ & $\begin{array}{l}\text { Downstaged }(n=330): 10 \text {-year } \\
\text { survival 52\%; } 10 \text {-year recurrence } 20 \% \\
\text { Milan criteria (no downstaging) } \\
\text { ( } n=2,086): 10 \text {-year survival 61\%; } \\
\text { 10-year recurrence } 14 \% \\
\text { Beyond Milan criteria, not down- } \\
\text { staged ( } n=110): 10 \text {-year survival } \\
39 \% ; 10 \text {-year recurrence } 47 \%\end{array}$ & $\begin{array}{l}p<0.001 \text { for beyond Milan } \\
\text { (not downstaged) vs. other } \\
\text { groups }\end{array}$ \\
\hline $\begin{array}{l}\text { Ravaioli 2008; } \\
\text { Bologna, Italy } \\
17\end{array}$ & $\begin{array}{l}\text { Bologna } \\
\text { criteria }\end{array}$ & $\begin{array}{l}\text { Prospective } \\
\text { single-center } \\
\text { study }\end{array}$ & $\begin{array}{l}\text { Downstaged }(n=32) \text { : 3-year tu- } \\
\text { mor-free survival } 71 \% \\
\quad \text { Milan criteria (no downstaging) } \\
(n=88): 3 \text {-year tumor-free survival } \\
71 \%\end{array}$ & NS \\
\hline
\end{tabular}

Abbreviation: UCSF, University of California San Francisco.

Note: UCSF downstaging criteria: 1 lesion $\leq 8 \mathrm{~cm} ; 2-3$ lesions $\leq 5 \mathrm{~cm}$ and total diameter $\leq 8 \mathrm{~cm} ; 4-5$ lesions $\leq 3 \mathrm{~cm}$ and total diameter $\leq 8 \mathrm{~cm}$. Bologna criteria: 1 lesion $\leq 6 \mathrm{~cm}$; 2 lesions $\leq 5 \mathrm{~cm}$; $3-5$ lesions $\leq 4 \mathrm{~cm}$ and total diameter $\leq 12 \mathrm{~cm}$. 


\section{Local Regional Therapy for Tumor Downstaging}

LRT for the purpose of downstaging of HCC to LT typically starts with a transarterial approach using conventional or drug-eluting bead transarterial chemoembolization (TACE), or yttrium-90 transarterial radioembolization (TARE). The majority of published series employed TACE as the mainstay of downstaging treatments. ${ }^{8,9}$ The advantages of using a transarterial approach upfront include complete targeting of large $(>5 \mathrm{~cm})$ lesions or of multiple lesions within a treated liver sector, and coverage of satellite nodules. Selection of the initial transarterial approach is dependent on the institutional expertise and remains a subject of considerable debate. The PREMIERE trial ${ }^{31}$ is a single-center randomized controlled trial comparing conventional TACE with TARE, and mainly focused on patients with HCC who were potentially eligible for bridging or downstaging to LT. Their results suggest a significantly longer median time to progression for patients who were treated with TARE ( $>26$ vs. 6.8 months), but the study is limited by the small number of patients in each arm. In a preliminary report from a large prospective multicenter study on downstaging involving seven centers (MERIT-LT consortium) $^{32}$ comparing 128 patients who underwent TACE and 61 patients who underwent TARE as initial downstaging treatment, there were no observed differences in mRECIST response, probability of downstaging, time to successful downstaging, or probability of waitlist dropout.

Underlying liver function is an important consideration when applying LRT for downstaging. The risk of hepatic decompensation or death is too high for a patient with Child's C cirrhosis (Child-Pugh-Turcotte score $\geq 10$ ) and total bilirubin $>3 \mathrm{mg} / \mathrm{dL}$ to justify the attempt for downstaging. ${ }^{8}$ In selected cases with mildly decompensated cirrhosis, however, several studies have assessed the use of TACE as a bridge to LT. ${ }^{33,34}$ These patients were found to be at an increased risk for treatment-related hepatic decompensation, but many were able to be treated successfully and safely using a superselective, subsegmental approach. TARE is typically reserved for patients with Child's A cirrhosis (Child-Pugh-Turcotte score 5-6) and serum total bilirubin $<2 \mathrm{mg} / \mathrm{dL}$. The generally accepted serum total bilirubin cutoff of $2 \mathrm{mg} / \mathrm{dL}$ for TARE is based on expert opinion $^{31,35}$ and the concern for increased risk of radioembolization-induced liver disease characterized by increasing total bilirubin and a sinusoidal obstruction syndrome among those with baseline total bilirubin $>2 \mathrm{mg} / \mathrm{dL}^{35}$ While TARE has been tried in some patients with mildly decompensated cirrhosis, ${ }^{36}$ more data on the safety and efficacy of TARE in this setting are needed.

Transarterial modalities may continue to be employed in the retreatment of residual or recurrent disease following the initial round of LRT, if underlying liver function permits safe LRT with the goal of maintaining patients within LT criteria. Lesions that develop extrahepatic arterial supply (e.g., via the phrenic, internal mammary, adrenal, omental, and intercostal arteries) may be safely treated with conventional TACE. There have been concerns about skin toxicity in using TACE with drug-eluting beads for tumors with extrahepatic supply due to the risk of deposition of permanent embolic particles within terminal arteries supplying the skin, whereas the experience with conventional TACE is extensive in this setting. ${ }^{37-39}$ TACE may be repeated multiple times provided that liver function remains adequate and as long as tumor-feeding arteries can be identified and catheterized. ${ }^{40}$ Repeated radiation segmentectomy targeting multiple different liver segments may be risky due to the expected irreversible radiation injury to the targeted liver segments and the risk of cumulative hepatotoxicity.

Alternative approaches for treatment of residual and/or recurrent tumors may include thermal ablation (radiofrequency or microwave), percutaneous ethanol injection, and external beam radiation or stereotactic body radiotherapy. Percutaneous thermal and chemical ablation should be limited to residual tumor nodules or satellite nodules up to $3 \mathrm{~cm}$ in maximal diameter. ${ }^{2,3}$ Tyrosine kinase inhibitors including sorafenib have not been shown to confer added benefits when used in combination with LRT as a bridge to $\mathrm{LT},{ }^{41}$ and have not been adequately studied in tumor downstaging prior to LT. In the past few years, several immunotherapies and small-molecule inhibitors have been approved for advanced HCC and are now being tested in combination with LRT. It remains unknown if these new systemic therapies have a role in pre-LT therapies among downstaging candidates.

\section{Tumor Staging after Local Regional Therapy}

Following transarterial therapy, disappearance of arterial hyperenhancement and washout with hypodense or hypointense lesion appearance on all phases of postcontrast imaging on CT or MRI is indicative of radiographic complete response based on mRECIST and EASL amendment. ${ }^{42}$ LI-RADS provides a detailed guidance on CT and MRI interpretation after LRT. ${ }^{43}$ Lack of residual enhancement or presence of treatment-specific enhancement pattern allows lesion categorization as LR-TR nonviable. Presence of nodular, mass-like, or thick irregular tissue in or along the lesion with (1) arterial phase hyperenhancement, or (2) washout appearance, or (3) enhancement similar to pretreatment is categorized as LR-TR viable. LR-TR equivocal response category is assigned for lesions demonstrating enhancement that is atypical for a treatment-specific expected enhancement pattern and not meeting criteria for probably or definitely viable. ${ }^{43}$

As already noted, radiographic assessments of tumor size following LRT should be based on measurements of the maximum diameter of only viable tumors by multiphase CT or MRI, and should not include the area of necrosis resulting from LRT. One problem that we encounter in this approach with downstaging to Milan as the end point is when there are more than one areas of viable tumor enhancement within the same nodule. For example, following LRT of a 6 -cm tumor, there are two areas of viable tumor within the same lesion each measuring $2 \mathrm{~cm}$ in maximal diameter (-Fig. 4). We do not recommend adding the diameter of these two areas (total maximal diameter of $4 \mathrm{~cm}$ ) or reclassifying the tumor as two separate tumors each $2 \mathrm{~cm}$ to render this as successful 


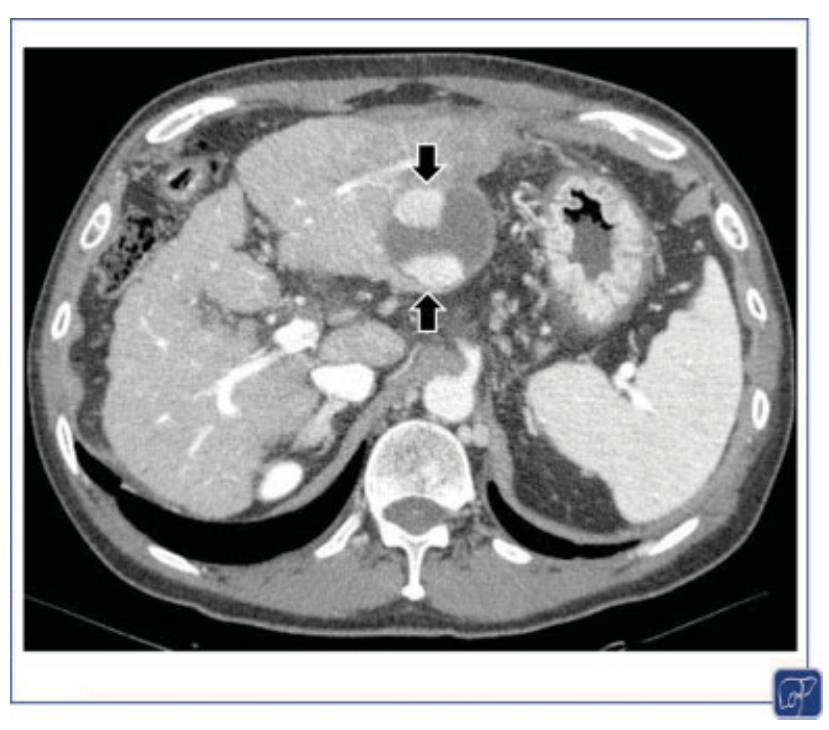

Fig. 4 Contrast-enhanced computed tomography images in the arterial phase illustrating two areas of tumor enhancement (arrows) within the tumor following transarterial chemoembolization with drug-eluting beads. (Modified with permission from Yao and Fidelman. ${ }^{8}$ )

downstaging to within Milan criteria. Instead, the tumor should be staged as a partially treated 6-cm tumor (LR-TR viable), and further treatments are needed for downstaging. If there is only one residual area of viable tumor measuring $2 \mathrm{~cm}$ after additional LRT, then the $2 \mathrm{~cm}$ viable tumor is now within Milan criteria and the tumor has been successfully downstaged.

After intra-arterial therapy. radiographic evaluation of treatment response is often not straightforward. Deposition of iodine-containing ethiodized oil within a lesion following conventional TACE may obscure areas of enhancing viable tumor on postprocedure CT (-Fig. 3), and incomplete deposition despite apparent complete response may be a sign of incomplete treatment. ${ }^{44}$ Under these circumstances, MRI may be helpful in identifying residual tumor. Changes within the target tissue perfusion on CT and MRI are common after embolotherapy. Postprocedure scans may show geographic regions of arterial hyperenhancement that may mimic residual or recurrent HCC. However, absence of delayed phase washout and restricted diffusion on MRI may help distinguish these parenchymal imaging alterations from residual or recurrent HCC ( - Fig. 5). These findings should be followed for stability or resolution at 2 to 3 month intervals. Evaluation of treatment response following TARE is much more challenging. Scans obtained fewer than 3 months after TARE often show residual arterial hyperenhancement within the treated lesions that is difficult to differentiate from residual viable tumor. ${ }^{45}$ Intra-arterial therapies deliver very high doses of potent therapeutic agents and may also cause tumor ischemia or hypoxia, resulting in acute necrosis and hemorrhage. These changes may even result in a paradoxical increase in tumor size at early follow-up imaging. In addition, treated tumors may demonstrate certain characteristic patterns of enhancement that may be mistaken for untreated or residual tumor. This enhancement may be secondary to posttreatment changes such as reactive edema or the forma- tion of granulation tissue. The use of quantitative diffusionweighted MRI or fluorine-18-fluorodeoxyglucose positron emission tomography ( ${ }^{18} \mathrm{~F}-\mathrm{FDG}$-PET) to address this problem requires further study using standardized protocols ${ }^{45}$. By 3 months after radiation, the tumors would be expected to decrease in size and to start developing typical imaging findings of necrosis. Some residual lesional hyperenhancement may persist for months after the treatment despite overall continued decrease of the lesion size. This problem in evaluating treatment response and presence of viable tumor may be even worse after external beam radiation or stereotactic body radiotherapy. For patients with high clinical suspicion of residual HCC due to interval lesional growth between the baseline and follow-up imaging studies and/or persistently elevated AFP, additional LRT with a different modality such as TACE or thermal ablation should be pursued without waiting 3 months after TARE.

While successful use of LRT for downstaging to LT criteria is adequate for activation on the LT list, continuation of LRT after successful downstaging until radiographic complete response by mRECIST is achieved is a common clinical practice at institutions with long wait times. Aggressive treatment of all residual and recurrent lesions using any available LRT modalities prevents disease progression and drop-out from the LT list. ${ }^{23}$ Patients with severely impaired liver function represent a notable exception. When enhancement at or near the treatment site is considered equivocal for HCC recurrence, close follow-up with repeat imaging in 2 to 3 months is needed to ensure stability and absence of radiologic features to suggest HCC recurrence. If tumor progression beyond LT criteria occurs but the active tumor burden remains within downstaging entry criteria, LT should be put on hold, and repeat LRT should be undertaken until the end point of downstaging is achieved for the patients to be eligible again for LT. ${ }^{8}$ Progression of tumors to beyond entry criteria for downstaging constitutes an exclusion criterion for LT (- Table $\mathbf{1}$ ).

\section{End Point of Downstaging}

The vast majority of published studies use tumor stage within Milan criteria on imaging as the end point of downstaging. ${ }^{8,9}$ Other studies have regarded downstaging as response to LRT based on the World Health Organization or mRECIST criteria. ${ }^{8}$ It seems logical to use the Milan criteria as the end point of downstaging, as this is in line with the liver allocation policy in the United States since 2002, granting listing priority to patients with HCC meeting the Milan criteria. Furthermore, the Milan criteria have been widely considered the benchmark against which the outcome using other criteria for LT as well as tumor downstaging should be measured. ${ }^{2-6}$ For further validation of the principles of downstaging, explant pathology should demonstrate a very low prevalence of unfavorable tumor characteristics including a poorly differentiated grade or microvascular invasion. These observations have been repeatedly demonstrated in studies using the UCSF downstaging inclusion criteria $^{23,25}$ but not in the Bologna study ${ }^{24}$. 


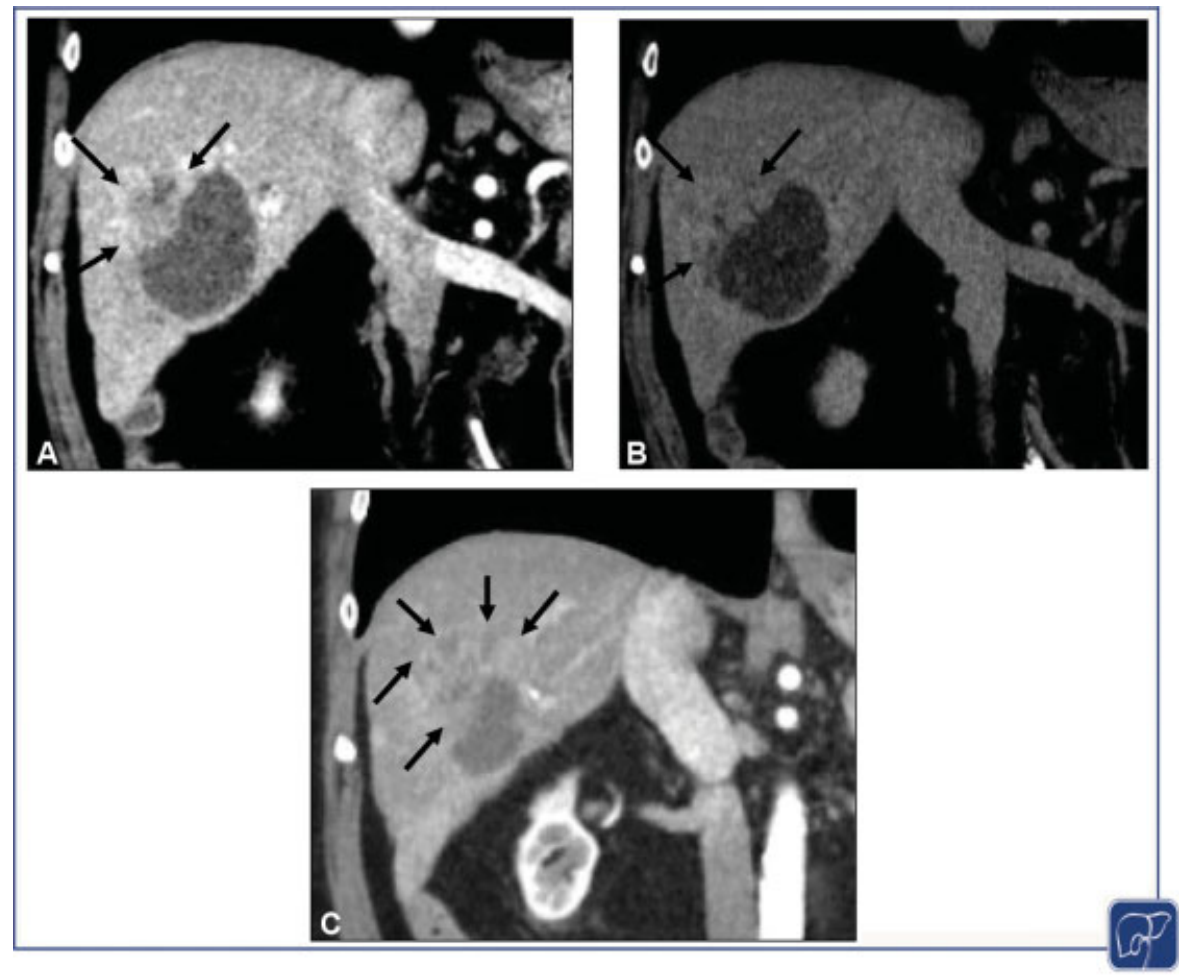

Fig. 5 Multiphase CT obtained 3 months after $\mathrm{Y}-90$ radioembolization showing extensive necrosis and an area of residual enhancement on the arterial phase at the superior aspect of the lesion (A, arrows) without washout on delayed phase (B, arrows). Follow-up imaging showed stability of this area of enhancement for 1 year, but a subsequent multiphase CT showed that the zone of arterial hyperenhancement had increased in size, with associated subtle washout consistent in appearance with HCC recurrence (C, arrows). CT, computed tomography.

Despite advances in cross-sectional imaging over the last few decades, radiographic understaging of HCC still occurs in 20 to $30 \%$ of patients with HCC classified as within Milan criteria by pre-LT imaging. ${ }^{4,46,47}$ Successful radiographic downstaging therefore does not necessarily assure actual downstaging based on explant pathology. It is possible that different imaging modalities may yield different rates of tumor understaging, but data on this are lacking. In studies on downstaging with well-defined upper limits of tumor size and number and a relatively long wait time from downstaging to LT, only $15 \%$ exceeded Milan criteria based on explant pathology (tumor understaging) ${ }^{23,25}$. Despite these encouraging results, tumor understaging remains a major concern with broader application of downstaging, when national data have continued to show sizable discrepancies between radiologic and explant pathologic staging. ${ }^{28,46,47}$ An analysis of the UNOS database reported that one-third of HCC patients initially meeting UCSF downstaging criteria had tumor beyond Milan criteria on explant, ${ }^{28}$ reflecting either pre-LT understaging or inadequate response to downstaging. Mahmud and colleagues recently reported the results of a comprehensive analysis of the UNOS/OPTN explant pathology form, in which tumor understaging in the explant was associated with increased post-LT HCC recurrence and death, and the risk of tumor-understaging was higher among those requiring tumor downstaging before LT. $^{47}$ Additionally, multiple explant-based prognostic models for estimation of post-LT HCC recurrence and surviv$\mathrm{al}^{48,49}$ have found that explant tumor beyond Milan portends worse post-LT prognosis. Further complicating matters, there have been concerns about inaccuracies and biases in reporting tumor size by LT centers, especially in patients outside of LT criteria who had their measurements recorded at the margins of LT eligibility. ${ }^{50}$ It has been shown that in patients who require tumor downstaging, the higher the tumor burden on the last imaging study prior to LT, the greater the risk of understaging on explant pathology. ${ }^{28}$ Specifically, the odds of tumor understaging on explant increases by $10 \%$ for each $1-\mathrm{cm}$ increase in total tumor diameter on the last pre-LT imaging study. ${ }^{28}$

Taken together, these findings highlight a very slim margin of error, and underscore the importance of strict adherence to downstaging definitions. To go one step further, we consider downstaging to within Milan criteria as the minimal requirement for LT, but advocate continued LRT until complete tumor necrosis is achieved in patients with good liver function to tolerate additional treatment. Although complete response to LRT by radiologic assessment does not guarantee the absence of viable tumor in the explant, there is strong evidence that achieving complete response to LRT with no viable tumor in the explant is associated with exceedingly low rates of post-LT HCC recurrence. ${ }^{48,51}$

\section{Incorporation of AFP and Other Factors in Downstaging Assessments}

With downstaging established as a standard pathway to LT, there are ongoing efforts to investigate other factors beyond 
tumor size and number that help identify subgroups with a greater likelihood of achieving successful downstaging and favorable post-LT outcome. Among surrogate markers of tumor biology, AFP is the most extensively studied and the most relevant. Elevated AFP is associated with increased probabilities of waitlist dropout and high-risk pathologic features including microvascular invasion, as well as worse post-LT outcome. ${ }^{52-54}$ Among patients who underwent tumor downstaging in the UNOS database, ${ }^{55}$ an initial AFP $>100$ or $>1,000 \mathrm{ng} / \mathrm{mL}$ was associated with a twoand fivefold greater risk of dropout, respectively, compared with those with an initial AFP $\leq 20 \mathrm{ng} / \mathrm{mL}$. While a reduction in the AFP following LRT predicts improved post-LT outcome when compared with those with persistently high AFP, ${ }^{56}$ assessing AFP response to LRT is difficult in patients with decompensated cirrhosis. These patients often have fewer LRT options or receive less aggressive treatments given the concerns for further hepatic decompensation, and have a higher risk of liver-related death irrespective of whether they receive LRT. In a multicenter study by Mehta et al using the UCSF downstaging protocol, ${ }^{25}$ treatment failure, defined as waitlist dropout, death without LT, or post-LT tumor recurrence, was observed in $100 \%$ of Child's B or C patients with initial AFP $\geq 1,000 \mathrm{ng} / \mathrm{mL}$. The authors concluded that these patients should be excluded from downstaging.

High AFP levels have also been consistently identified as a negative predictor of post-LT outcome independent of tumor burden. $^{12,13,28,48,53,54}$ Both the French AFP model ${ }^{12}$ and the Metroticket $2.0^{13}$ have demonstrated that a composite of AFP and tumor burden parameters discriminates post-LT prognosis significantly better than tumor burden alone. Additionally, rising AFP despite LRT while awaiting LT appears to an indicator of poor prognosis. ${ }^{57,58}$ In the downstaging population, AFP $>100 \mathrm{ng} / \mathrm{mL}$ at the time of LT predicts higher risks of HCC recurrence and death, with a 3-year post-LT survival of 60 versus $81 \%$ for those with an AFP $<20 \mathrm{ng} / \mathrm{mL}^{28}$ Liver allocation policy in the United States now mandates an AFP cutoff of $<500 \mathrm{ng} / \mathrm{mL}$ after LRT in patients with AFP ever $>1,000 \mathrm{ng} / \mathrm{mL}$ to be eligible for $\mathrm{LT}^{30,53}$ This has since been validated using the UNOS database, in which the 5-year post-LT survival for those with AFP $>1,000$ at LT was 49 versus $67 \%$ for those with a decrease in AFP to 101 to 499 and $88 \%$ for those with a further decrease in AFP to $\leq 100$ before LT. $^{56}$ For patients undergoing downstaging, we propose that those with initial AFP $>1,000 \mathrm{ng} / \mathrm{mL}$ should meet a stricter AFP cutoff goal of $\leq 100 \mathrm{ng} / \mathrm{mL}$ after LRT to be eligible for LT.

Apart from AFP, several other serum biomarkers have been studied in relation to HCC recurrence after LT. An elevated AFPL3 of $>35 \%$ and des- $\gamma$ carboxy prothrombin (DCP) of $>7.5 \mathrm{ng} / \mathrm{mL}$ have been shown to correlate with worse post-LT outcomes, ${ }^{59-61}$ but the significance of these biomarkers in downstaging patients is largely unknown. ${ }^{18} \mathrm{~F}$-FDG-PET tumor positivity, especially if the tumor-to-nontumor ratio is $\geq 2$, has been associated with worse recurrence-free survival after live-donor LT. $^{62}$ PET-positive patients with elevated AFP appear to have particularly poor post-LT outcome. ${ }^{63}$ While the potential role for PET imaging in downstaging candidates is unknown, PET can be considered on an individualized basis, particularly if the anticipated wait time to LT after downstaging is short, if living donor LT is an option, or in the situation of persistently elevated or rising AFP.

Lastly, a short LT wait time of under 4 to 6 months has been linked to worse post-LT survival. ${ }^{64,65}$ For patients undergoing tumor downstaging, a minimal observation period of 3 months from successful downstaging by radiologic criteria to LT without additional LRT has been proposed to adequately assess disease stability and to avoid transplanting aggressive tumors. ${ }^{8,16}$ In a recent analysis of national data on tumor downstaging prior to LT by Mehta et al, ${ }^{28}$ patients from regions with a relatively short wait time of $<6$ months had worse 3 -year post-LT survival of $<80 \%$ compared with $>90 \%$ for those in longer wait regions with a median wait time of 13 months. These results suggest that in downstaging of HCC prior to LT, there is at least a theoretical benefit in allowing sufficient time to select the more appropriate candidates for LT.

\section{Conclusion}

It was 10 years ago when the International Consensus Conference on LT for HCC set the high expectation for tumor downstaging to achieve comparable post-LT survival between patients who achieve successful tumor downstaging before LT and those whose tumors meet LT criteria at the outset without needing downstaging. ${ }^{66}$ Since that time, efforts have been made to validate eligibility criteria based on initial tumor burden and refine LT candidate selection using biomarkers such as AFP, and to produce outcome data suggesting that this goal is indeed attainable. New challenges emerge as we have entered the next phase of implementing downstaging at a national level to allow priority listing for LT for those meeting criteria for successful tumor downstaging. Ironically, tumor "understaging" has surfaced as a major problem to overcome with tumor downstaging. More aggressive LRT until complete tumor necrosis in patients with adequate hepatic reserve and using stringent AFP thresholds may lower the rate of tumor understaging. Most importantly, applying validated radiologic criteria in HCC diagnosis and assessment of response to LRT and strict adherence to staging definitions for inclusion and end points of downstaging are essential in optimizing the results of downstaging as well as post-LT outcomes.

\section{Main Concepts and Learning Points}

- Downstaging is defined as a reduction in the size of viable tumors using local-regional therapy to meet acceptable criteria for liver transplant and serves as a selection tool for a subgroup of HCC patients with favorable tumor biology.

- The goal of downstaging is to achieve similar post-transplant survival between patients who achieve successful tumor downstaging before transplant and patients initially within conventional transplant criteria without needing downstaging. 
- Applying validated radiologic criteria in HCC diagnosis and assessment of response to local-regional therapy with strict adherence to staging definitions for inclusion and end points of downstaging are essential in optimizing the results of downstaging including post-transplant outcomes.

- Tumor "understaging" has surfaced as a significant problem with the recommendation for aggressive local-regional therapy until complete tumor necrosis in patients with adequate hepatic reserve and using more stringent AFP thresholds to lower the rate of tumor understaging.

\section{Conflict of Interest}

Dr. Yao reports grants from Fujifilm Wako, other from Gilead Sciences, outside the submitted work. Dr. Fidelman reports grants from Merck, grants from Boston Scientific, grants from Sirtex Medical, outside the submitted work. Dr. Mehta reports grants and other from Fujifilm Wako, grants from Glycotest, grants from Target Pharmasolutions, outside the submitted work.

\section{References}

1 Mazzaferro V, Regalia E, Doci R, et al. Liver transplantation for the treatment of small hepatocellular carcinomas in patients with cirrhosis. N Engl J Med 1996;334(11):693-699

2 Bruix J, Sherman MPractice Guidelines Committee, American Association for the Study of Liver Diseases. Management of hepatocellular carcinoma. Hepatology 2005;42(05):1208-1236

3 European Association for the Study of the Liver. Electronic address: easloffice@easloffice.eu European Association for the Study of the Liver. EASL clinical practice guidelines: management of hepatocellular carcinoma. J Hepatol 2018;69(01):182-236

4 Verna EC, Patel YA, Aggarwal A, et al. Liver transplantation for hepatocellular carcinoma: management after the transplant. Am J Transplant 2020;20(02):333-347

5 Mehta N, Bhangui P, Yao FY, et al. Liver transplantation for hepatocellular carcinoma. Working group report from the ILTS Transplant Oncology Consensus Conference. Transplantation 2020;104(06):1136-1142

6 Mazzaferro V. Squaring the circle of selection and allocation in liver transplantation for HCC: an adaptive approach. Hepatology 2016;63(05):1707-1717

7 Mehta N, Yao FY. Moving past "one size (and number) fits all" in the selection of candidates with hepatocellular carcinoma for liver transplantation. Liver Transpl 2013;19(10):1055-1058

8 Yao FY, Fidelman N. Reassessing the boundaries of liver transplantation for hepatocellular carcinoma: where do we stand with tumor down-staging? Hepatology 2016;63(03):1014-1025

9 Parikh ND, Waljee AK, Singal AG. Downstaging hepatocellular carcinoma: a systematic review and pooled analysis. Liver Transpl 2015;21(09):1142-1152

10 Yao FY, Ferrell L, Bass NM, et al. Liver transplantation for hepatocellular carcinoma: expansion of the tumor size limits does not adversely impact survival. Hepatology 2001;33(06):1394-1403

11 Mazzaferro V, Llovet JM, Miceli RMetroticket Investigator Study Group. , et al; . Predicting survival after liver transplantation in patients with hepatocellular carcinoma beyond the Milan criteria: a retrospective, exploratory analysis. Lancet Oncol 2009;10 (01):35-43

12 Duvoux C, Roudot-Thoraval F, Decaens TLiver Transplantation French Study Group. et al;. Liver transplantation for hepatocellular carcinoma: a model including $\alpha$-fetoprotein improves the performance of Milan criteria. Gastroenterology 2012;143(04): 986-94.e3, quiz e14-e15
13 Mazzaferro V, Sposito C, Zhou J, et al. Metroticket 2.0 model for analysis of competing risks of death after liver transplantation for hepatocellular carcinoma. Gastroenterology 2018;154(01):128-139

14 Toso C, Meeberg G, Hernandez-Alejandro R, et al. Total tumor volume and alpha-fetoprotein for selection of transplant candidates with hepatocellular carcinoma: a prospective validation. Hepatology 2015;62(01):158-165

15 Sapisochin G, Goldaracena N, Laurence JM, et al. The extended Toronto criteria for liver transplantation in patients with hepatocellular carcinoma: a prospective validation study. Hepatology 2016;64(06):2077-2088

16 Roberts JP, Venook A, Kerlan R, Yao F. Hepatocellular carcinoma: ablate and wait versus rapid transplantation. Liver Transpl 2010; 16(08):925-929

17 Mitchell DG, Bruix J, Sherman M, Sirlin CB. LI-RADS (Liver Imaging Reporting and Data System): summary, discussion, and consensus of the LI-RADS Management Working Group and future directions. Hepatology 2015;61(03):1056-1065

18 Wald C, Russo MW, Heimbach JK, Hussain HK, Pomfret EA, Bruix J. New OPTN/UNOS policy for liver transplant allocation: standardization of liver imaging, diagnosis, classification, and reporting of hepatocellular carcinoma. Radiology 2013;266(02):376-382

19 Palmer WC, Patel T. Are common factors involved in the pathogenesis of primary liver cancers? A meta-analysis of risk factors for intrahepatic cholangiocarcinoma. J Hepatol 2012;57(01):69-76

20 Sapisochin G, Fidelman N, Roberts JP, Yao FY. Mixed hepatocellular cholangiocarcinoma and intrahepatic cholangiocarcinoma in patients undergoing transplantation for hepatocellular carcinoma. Liver Transpl 2011;17:934-942

21 Lencioni R, Llovet JM. Modified RECIST (mRECIST) assessment for hepatocellular carcinoma. Semin Liver Dis 2010;30(01):52-60

22 Reig M, Darnell A, Forner A, Rimola J, Ayuso C, Bruix J. Systemic therapy for hepatocellular carcinoma: the issue of treatment stage migration and registration of progression using the BCLCrefined RECIST. Semin Liver Dis 2014;34(04):444-455

23 Yao FY, Mehta N, Flemming J, et al. Downstaging of hepatocellular cancer before liver transplant: long-term outcome compared to tumors within Milan criteria. Hepatology 2015;61(06):1968-1977

24 Ravaioli M, Grazi GL, Piscaglia F, et al. Liver transplantation for hepatocellular carcinoma: results of down-staging in patients initially outside the Milan selection criteria. Am J Transplant 2008;8(12):2547-2557

25 Mehta N, Guy J, Frenette CT, et al. Excellent outcomes of liver transplantation following down-staging of hepatocellular carcinoma to within Milan criteria: a multicenter study. Clin Gastroenterol Hepatol 2018;16(06):955-964

26 Murali AR, Romero-Marrero C, Miller C, et al. Predictors of successful downstaging of hepatocellular carcinoma outside Milan criteria. Transplantation 2016;100(11):2391-2397

27 Sinha J, Mehta N, Dodge JL, Poltavskiy E, Roberts JP, Yao FY. Are there upper limits in tumor burden for down-staging of hepatocellular carcinoma to liver transplant? Analysis of the all-comers protocol. Hepatology 2019;70:1185-1196

28 Mehta N, Dodge JL, Grab JD, Yao FY. National experience on downstaging of hepatocellular carcinoma before liver transplant: influence of tumor burden, alpha-fetoprotein, and wait time. Hepatology 2020;71(03):943-954

29 Tabrizian P, Holzner M, Halazun K, et al. A US multicenter analysis of 2529 HCC patients undergoing liver transplantation: 10-year outcome assessing the role of downstaging to within Milan criteria. . [Abstract]Hepatology 2019;70:11A

30 OPTN/UNOS Liver and Intestinal Organ Transplantation Committee. Organ Procurement and Transplantation Network. Available at: http://optn.transplant.hrsa.gov/. Accessed May 10, 2020

31 Salem R, Gordon AC, Mouli S, et al. Y90 radioembolization significantly prolongs time to progression compared with chemoembolization in patients with hepatocellular carcinoma. Gastroenterology 2016;151(06):1155.e2-1163.e2 
32 Mehta N, Guy J, Frenette C, et al Transarterial chemoembolization and radio-embolization are similarly efficacious in achieving successful hepatocellular carcinoma (HCC) down-staging: results from the Multicenter Evaluation of Reduction in Tumor Size Before Liver Transplantation (MERITS-LT) Consortium [Abstract]. Paper presented at: American Transplant Congress 2020; May 30 to June 3, 2020; Philadelphia, PA

33 Garwood ER, Fidelman N, Hoch SE, Kerlan RK Jr, Yao FY. Morbidity and mortality following transarterial liver chemoembolization in patients with hepatocellular carcinoma and synthetic hepatic dysfunction. Liver Transpl 2013;19(02):164-173

34 Fidelman N, Johanson C, Kohi MP, et al. Prospective phase II trial of drug-eluting bead chemoembolization for liver transplant candidates with hepatocellular carcinoma and marginal hepatic reserve. J Hepatocell Carcinoma 2019;6:93-103

35 Sangro B, Martínez-Urbistondo D, Bester L, et al. Prevention and treatment of complications of selective internal radiation therapy: expert guidance and systematic review. Hepatology 2017;66(03): 969-982

36 Uddin O, Gabr A, Abouchaleh N, et al. Radioembolization for hepatocellular carcinoma in patients with hyperbilirubinemia. . [Abstract]J Vasc Interv Radiol 2017;28:S74-S75

37 Miyayama S, Matsui O, Taki K, et al. Extrahepatic blood supply to hepatocellular carcinoma: angiographic demonstration and transcatheter arterial chemoembolization. Cardiovasc Intervent Radiol 2006;29(01):39-48

38 Li Q Ao GK, Duan F, Wang ZJ, Yan JY, Wang MQ. Incidence and therapeutic frequency of extrahepatic collateral arteries in transcatheter arterial chemoembolization of hepatocellular carcinoma: Experience from 182 patients with survival time more than 3 years. Eur J Radiol 2015;84(12):2555-2563

39 Lokken RP, Fidelman N, Kolli KP, Kerlan RK Jr. Safety and efficacy of doxorubicin drug-eluting embolic chemoembolization of hepatocellular carcinoma supplied by extrahepatic collateral arteries. J Vasc Interv Radiol 2016;27(11):1698-1704

40 Sieghart W, Hucke F, Pinter M, et al. The ART of decision making: retreatment with transarterial chemoembolization in patients with hepatocellular carcinoma. Hepatology 2013;57(06):2261-2273

41 Hoffmann K, Ganten T, Gotthardtp D, et al. Impact of neo-adjuvant Sorafenib treatment on liver transplantation in HCC patients - a prospective, randomized, double-blind, phase III trial. BMC Cancer 2015; $15: 392$

42 Gordic S, Corcuera-Solano I, Stueck A, et al. Evaluation of HCC response to locoregional therapy: validation of MRI-based response criteria versus explant pathology. J Hepatol 2017;67(06): 1213-1221

43 American College of Radiology CT/MRI LIRADS v. 2018, Available at: https://www.acr.org/-/media/ACR/Files/RADS/LI-RADS/LI-RADS2018-Core.pdf?la=en. Accessed May 10, 2020

44 Dioguardi Burgio M, Ronot M, Bruno O, et al. Correlation of tumor response on computed tomography with pathological necrosis in hepatocellular carcinoma treated by chemoembolization before liver transplantation. Liver Transpl 2016;22(11):1491-1500

45 Guo Y, Yaghmai V, Salem R, et al. Imaging tumor response following liver-directed intra-arterial therapy. Abdom Imaging 2013;38(06):1286-1299

46 Harper AM, Edwards E, Washburn WK, Heimbach J. An early look at the Organ Procurement and Transplantation Network explant pathology form data. Liver Transpl 2016;22(06):757-764

47 Mahmud N, Hoteit MA, Goldberg DS. Risk factors and center-level variation in hepatocellular carcinoma under-staging for liver transplantation. Liver Transpl 2020; 26(08):977-988

48 Mehta N, Heimbach J, Harnois DM, et al. Validation of a risk estimation of tumor recurrence after transplant (RETREAT) score for hepatocellular carcinoma recurrence after liver transplant. JAMA Oncol 2017;3(04):493-500

49 Agopian VG, Harlander-Locke M, Zarrinpar A, et al. A novel prognostic nomogram accurately predicts hepatocellular carcinoma recur- rence after liver transplantation: analysis of 865 consecutive liver transplant recipients. J Am Coll Surg 2015;220(04):416-427

50 Samoylova ML, Nigrini MJ, Dodge JL, Roberts JP. Biases in the reporting of hepatocellular carcinoma tumor sizes on the liver transplant waiting list. Hepatology 2017;66(04):1144-1150

51 Agopian VG, Morshedi MM, McWilliams J, et al. Complete pathologic response to pretransplant locoregional therapy for hepatocellular carcinoma defines cancer cure after liver transplantation: analysis of 501 consecutively treated patients. Ann Surg 2015;262 (03):536-545, discussion 543-545

52 Mehta N, Dodge JL, Hirose R, Roberts JP, Yao FY. Predictors of low risk for dropout from the liver transplant waiting list for hepatocellular carcinoma in long wait time regions: Implications for organ allocation. Am J Transplant 2019;19(08):2210-2218

53 Hameed B, Mehta N, Sapisochin G, Roberts JP, Yao FY. Alphafetoprotein level $>1000 \mathrm{ng} / \mathrm{mL}$ as an exclusion criterion for liver transplantation in patients with hepatocellular carcinoma meeting the Milan criteria. Liver Transpl 2014;20(08):945-951

54 Berry K, Ioannou GN. Comparison of liver transplant-related survival benefit in patients with versus without hepatocellular carcinoma in the United States. Gastroenterology 2015;149(03): 669-680, quiz e15-e16

55 Huang A, Dodge J, Yao F, Mehta N. National experience on waitlist outcomes for down-staging of hepatocellular carcinoma to within Milan criteria: high dropout rate in the "all-comers" group. . [Abstract]Hepatology 2019;70(S1):90A

56 Mehta N, Dodge JL, Roberts JP, Hirose R, Yao FY. Alpha-fetoprotein decrease from $>1,000$ to $<500 \mathrm{ng} / \mathrm{mL}$ in patients with hepatocellular carcinoma leads to improved posttransplant outcomes. Hepatology 2019;69(03):1193-1205

57 Lai Q Inostroza M, Rico Juri JM, Goffette P, Lerut J. Delta-slope of alpha-fetoprotein improves the ability to select liver transplant patients with hepatocellular cancer. HPB (Oxford) 2015;17(12): 1085-1095

58 Giard JM, Mehta N, Dodge JL, Roberts JP, Yao FY. Alpha-fetoprotein slope $>7.5 \mathrm{ng} / \mathrm{mL}$ per month predicts microvascular invasion and tumor recurrence after liver transplantation for hepatocellular carcinoma. Transplantation 2018;102(05):816-822

59 Chaiteerakij R, Zhang X, Addissie BD, et al. Combinations of biomarkers and Milan criteria for predicting hepatocellular carcinoma recurrence after liver transplantation. Liver Transpl 2015; 21(05):599-606

60 Kaido T, Ogawa K, Mori A, et al. Usefulness of the Kyoto criteria as expanded selection criteria for liver transplantation for hepatocellular carcinoma. Surgery 2013;154(05):1053-1060

61 Lee JH, Cho Y, Kim HY, et al. Serum tumor markers provide refined prognostication in selecting liver transplantation candidate for hepatocellular carcinoma patients beyond the Milan criteria. Ann Surg 2016;263(05):842-850

62 Hsu CC, Chen CL, Wang CC, et al. Combination of FDG-PET and UCSF criteria for predicting HCC recurrence after living donor liver transplantation. Transplantation 2016;100(09):1925-1932

63 Hong G, Suh KS, Suh SW, et al. Alpha-fetoprotein and (18)F-FDG positron emission tomography predict tumor recurrence better than Milan criteria in living donor liver transplantation. J Hepatol 2016;64:852-859

64 Halazun KJ, Patzer RE, Rana AA, et al. Standing the test of time: outcomes of a decade of prioritizing patients with hepatocellular carcinoma, results of the UNOS natural geographic experiment. Hepatology 2014;60(06):1957-1962

65 Mehta N, Heimbach J, Lee D, et al. Wait time of less than 6 and greater than 18 months predicts hepatocellular carcinoma recurrence after liver transplantation: proposing a wait Time "sweet spot". Transplantation 2017;101(09):2071-2078

66 Clavien PA, Lesurtel M, Bossuyt PM, Gores GJ, Langer B, Perrier AOLT for HCC Consensus Group. Recommendations for liver transplantation for hepatocellular carcinoma: an international consensus conference report. Lancet Oncol 2012;13(01):e11-e22 